\title{
GASTRIC CANCER TUMOR MARKERS
}

\author{
Alexandra Gireada ${ }^{1}$, Irina Balescu ${ }^{2}$, Nicolae Bacalbasa ${ }^{3}$ \\ 1 "Carol Davila" Central Military Emergency Hospital, Bucharest, Romania \\ 2 "Ponderas" Hospital, Bucharest, Romania \\ 3 "Carol Davila" University of Medicine and Pharmacy, Bucharest, Romania
}

\begin{abstract}
Gastric cancer remains an aggressive malignancy responsible for a high number of deaths annually. An important method used in order to determine the response to treatment, follow-up and detection of recurrence in association with imagistic examinations remains laboratory determination of tumor markers. The most common used tumor markers in patients diagnosed with gastric cancer are CA 72-4, CEA, CA 19-9.
\end{abstract}

Keywords: gastric cancer, CA 72-4, CEA, CA 19-9

\section{INTRODUCTION}

Gastric cancer is the third malignancy in the world in terms of mortality, mainly because it is diagnosed in an advanced stage. Once known as the second most common cancer in the world, its frequency has declined over the past half century due to the increased consumption of fresh fruits and vegetables, decreased intake of salt, the refrigeration of meat products and the discovery of antibiotics against Helicobacter pylori. (1)

The laboratory investigations most frequently used in patients with gastric cancer are the complete blood count, $30 \%$ of patients being diagnosed with anemia (caused by bleeding, liver disfunction and poor ferum intake), the electrolyte panels, the liver function tests and the tumor markers. (1)

The international guidelines established the utility of the tumor markers in the gastric cancer. They are used for monitoring the cytostatic treatment (but the gold standard is considered radiology), for the detection of recurrences (although this doesn't lead to the improvement of survival rates) and they are not accepted for diagnostic purposes. (2)

The most utilized tumor markers in gastric cancer are: CA 72-4, CEA and CA 19-9 (3).

\section{CA 72-4}

\section{The role of $C A$ 72-4 in the diagnosis}

CA 72-4 is a glycoprotein which can be detected in gastric, pancreatic, colorectal, ovarian and breast cancers. $(4,5)$ Its normal concentrations are lower than $6.9 \mathrm{U} / \mathrm{mL}$. (6) Literature data show a sensitivity of $40 \%$ in the detection of gastric cancer, of $40 \%$ in the detection of colorectal cancer, $50 \%$ in the detection of ovarian cancer and an overall specificity of $95 \%$. (7) A meta-analysis realized by Chen on the bases of 33 studies proved that CA 72-4 was the tumor marker with the highest overall accuracy $(77 \%)$ in the detection of gastric cancer. (8)

\section{The role of $C A$ 72-4 in estimating prognosis}

Research results have proved some correlations between the concentrations of CA 72-4 and the stage of lymph node involvement and the tumor resectability. (9-11) In a study realized by Safi (Department of General Surgery, University of Ulm, Germany), the results showed a good correlation between the CA 72-4 concentrations and tumor stage. (12) 


\section{CEA}

\section{The role of CEA in estimating prognosis}

CEA is a glycoprotein with a half-life of three days. This gives the possibility of determining the concentrations every 7 days. Literature data show that there is a correlation between the increased pre-treatment concentrations of CEA and the stage of the disease. (13) A recent study showed a correlation between the pre-treatment CEA values and the TNM stage, lymph node invasion and the T category. (14) Other research results show that the normal pre-treatment concentrations are associated with a good survival. (15) It was also found that the presence of normal postoperative concentrations of CEA over a period of less than 2 months is associated with a food overall survival. $(16,17)$ The studies showed that the CEA levels are higher and that the pathological values are more frequently encountered in young patients (aged between 18 and 30 years old). These patients are often characterized by greater tumor sizes and lymph-vascular involvement and thus by a worse overall survival. (18)

\section{The role of $C E A$ in the diagnosis}

Statistical data show that CEA has a sensitivity of $30 \%$ in the detection of gastric cancer and a negative predictive value of $58.82 \%$. The values found for the colorectal cancer are higher $(74 \%$ and $79.36 \%$, respectively). (19)

\section{The role of $C E A$ in the detection of recurrences}

Other studies found a statistically significant correlation between the serum CEA concentrations and locoregional relapse. $(20,21)$ The CEA determination can be useful for the detection of liver metastases recurrences, which can precede the radiological detection of the relapse by over 3 months. (22) Literature data prove the association of CEA increase with relapse, with the highest sensitivity attained in the case of peritoneal involvement. $(23,24)$

\section{CA 19-9}

CA 19-9 is a protein with normal concentrations of $<37 \mathrm{UI} / \mathrm{L}$ and a half-life of 1 to 3 days (25).

\section{The role of $C A$ 19-9 in the diagnosis}

CA $19-9$ has a sensitivity of $42 \%$ and a negative predictive value of $58.82 \%$ in gastric cancer. In colorectal cancer, the values are of only $26 \%$ and $57.47 \%$, respectively. (19)

\section{The role of $C A$ 19-9 in determining prognosis}

The determination of CA 19-9 may have a prognostic significance, since the preoperative values are correlated with the stage of the disease and the persistence of elevated levels for more than 2 months after the surgical treatment indicates a guarded prognosis. $(11,17,26-28)$. Research results showed a correlation with the lymph node involvement (29). A recent study showed a correlation between the pre-treatment CEA values and the TNM stage, lymph node invasion and the T category (30).

\section{The role of $C A 19-9$ in the detection of recurrences}

CA 19-9 can be also used for the detection of recurrences. It can detect them with over 2 months earlier than the radiological method. (21)

\section{THE COMPARISON BETWEEN VARIOUS SERUM MARKERS}

\section{The value of various tumor markers in the diagnosis}

A study realized by D.J. Byrne et al analyzed patients with gastric cancer and the role of the tumor markers CA 72-4, CEA and CA 19-9 in their management. The best marker that could separate patients diagnosed with the first stages of the disease from control subjects was CA 72-4. Only CA 72-4 and CEA could differentiate between patients with positive and negative nodes. Only CA 72-4 assay could differentiate between serosa positive and serosa negative patients. CA $72-4$ had the highest sensitivity at a given specificity. The conclusion of the study was that CA 72-4 can be successfully used for estimating the disease stage and activity in gastric cancer, although more research is still necessary. (31)

A study realized by Heptner in 1989 compared the role of several tumor markers (CA72-4, CA 19-9 and CEA) in the diagnosis of gastric cancer. The sensitivity of CA $72-4$ was of $59 \%$, the sensitivity of CA $19-9$ was $52 \%$ and the sensitivity of CEA was $70 \%$. The results showed an increased specificity of CA $72-4$ (98\%) in the detection of benign (even inflammatory) conditions of the gastrointestinal tract (32).

Another study realized by Joypaul in 1993 included 52 patients with gastric adenocarcinomas and 32 patients with benign gastric disorders. Its results found a sensitivity of $42 \%$ for CA $72-4$, of $46 \%$ for CA $19-9$ and of $63 \%$ for the association of the two tumor markers for the detection of gastric cancer. The specificity of CA $72-4(100 \%)$ was higher than that of CA 19-9 (only 72\%). (33) 


\section{The value of various tumor markers in estimating prognosis}

A study realized by A. Mittal et al in 2012 intended to find out the value of the tumor markers in estimating the prognosis of gastric cancer. It included 40 patients with tumors localized in different regions of the stomach and being characterized by different lymph node stages. The authors measured the serum concentrations of AFP, CEA, CA19-9 and CA50 by means of the ELISA method. The study used a cut-off value of $10 \mu \mathrm{g} / \mathrm{l}$ for AFP, $10 \mu \mathrm{g} / 1$ for CEA, $37 \mathrm{U} / \mathrm{ml}$ for CA19-9, and $20 \mathrm{U} / \mathrm{ml}$ for CA50. The results showed that CEA, CA19-9, CA50, tumor size and lymph node stage can be considered independent prognostic factors in gastric cancer. These tumor markers proved to detect especially the advanced stages of gastric cancer, their concentrations can predict the patients' prognosis and their preoperative rises are the sign of a poor prognosis and of the necessity of adjuvant management. (34)

A study realized in 2011 evaluated the predictive value of for tumor markers : CEA (cut-off $=5 \mathrm{ng} / \mathrm{ml}$ ), CA 19-9 (cut-off $=37 \mathrm{U} / \mathrm{ml})$, CA $72-4$ (cut-off $=4 \mathrm{U} /$ $\mathrm{ml}$ ) and $\beta$-hCG (cut-off $=5 \mathrm{UI} / \mathrm{ml}$ ) for preoperative staging in gastric cancer. The results showed that the preoperative concentration of CA 72-4 had the highest accuracy in the detection of advanced disease. An even higher accuracy is attained by the four marker combination. The concentrations of these tumor markers were not correlated with histological type or tumoral grade. (35)

A study realized by D.H. Nam et al investigated the prognostic significance of postoperative values of different tumor markers in gastric cancer. The study included 206 patients treated by radical gastrectomy between 2001 and 2007. All these patients had increased CEA concentrations $(>5 \mathrm{ng} / \mathrm{ml})$ and increased CA 19-9 concentrations $(>37 \mathrm{U} / \mathrm{mL})$ before treatment. The authors defined early tumor marker response as the normalization of CEA and CA 19-9 at 1-2 months after gastrectomy. The results showed that $72.8 \%$ of the patients achieved early tumor marker response. They were characterized by longer disease-free survival and overall survival. (17)

A study conducted by X. Liu et al included 273 patients diagnosed with $\mathrm{T} 4 \mathrm{a}$ gastric cancer and treated by curative gastrectomy. The authors measured the concentrations of AFP, CEA, CA 19-9 and CA50 and evaluated their prognostic role. The results showed good correlations between AFP and Borrmann type, between CEA and sex, tumors site, and $\mathrm{N}$ stage, between CA 19-9 and age, tumor site, lymphovascular invasion and $\mathrm{N}$ stage. The increased concentrations of CEA, CA 19-9 and CA 50 were found to be independent poor prognostic factors for these patients in multivariate analysis. (36)

\section{The role of various tumor markers in the detection of recurrences}

In a study realized at Safi (Department of General Surgery, University of Ulm, Germany), the sensitivity of CA 72-4 was greater than that of CEA and CA 19-9 in the detection of gastric cancer recurrences. (12)

A study realized by Marrelli found a sensitivity of $44 \%$ for CEA in the detection of recurrences, of $56 \%$ for CA $19-9$ and of $51 \%$ for CA $72-4$. (37)

A study effectuated by J.H. Yook et al included 170 gastric cancer patients. Their preoperative and postoperative concentrations of CEA, CA 19-9 and CA 72-4 were measured and the sensitivities in the detection of recurrences were $44 \%, 21 \%$ and $21 \%$, respectively. The association of CEA with CA 19-9 or CA 72-4 led to a sensitivity of 58\%. CEA had the highest sensitivity rate in detecting recurrences at the anastomotic site, CA 19-9 in detecting recurrences of lymph node, CA 72-4 in detecting recurrences at peritoneal seeding and distant metastasis. (38)

\section{The role of various tumor markers in monitoring treatment}

A study realized by T. Yamao et al included 26 patients diagnosed with gastric cancer, in an advanced stage, with increased concentrations of CEA, CA 19-9 and CA 125 before systemic chemotherapy. A response to treatment was considered as $a \geq 50 \%$ decrease in the concentration maintained for more than 4 weeks. The results showed a good correlation between the imaging studies and the assessment of response by tumor markers. The patients who responded to treatment were also characterized by longer survival times. The conclusions of the study were that tumor markers can be useful in monitoring the response to treatment and in estimating the prognosis of patients with gastric cancer. (39)

\section{OTHER GASTRIC TUMOR MARKERS}

VEGF is considered to be an independent prognostic factor in patients with gastric carcinoma. It is also useful in the detection of liver cancer recurrence. Another marker is c-erbB-2, which corre- 
lates with tissue overexpression of HER-2/neu. Increased MMP-9 concentrations correlate with short survival rates and with stromal reaction. Increased concentrations of SICAM are associated with hematogenous metastasis and with a poor prognosis. The plasma levels of TIMP-1 are considered to be independent and powerful prognostic factors for the survival of patients. Increased plasma VEGF levels are found in patients with liver metastasis. (40)

A study realized in 2013 included 363 gastric cancer patients. The authors, S Fujiwara et al measured the concentration of NY-ESO-1 antibody in the serum of these patients by means of the ELISA method. This antibody is characteristic for patients having tumors that express the NY-ESO-1 antigen. $3.4 \%$ of patients with stage I disease, $4.4 \%$ of pa- tients with stage II disease, $25.3 \%$ of patients with stage III disease and $20 \%$ of patients with stage IV disease had increased serum levels of NY-ESO-1 antigen. These sensitivities were increased by the use of the combination of NY-ESO-1 antibody, CEA and CA 19-9. Thus the conclusion of the study was that the combination of markers can be useful in the detection of advanced stages of the gastric cancer and in estimating treatment response. (41)

\section{CONCLUSIONS}

Available literature data show that the discovery of new tumor markers and the combinations of tumor markers are the best solutions for the improvement of patients' management, but more research is still necessary.

\section{REFERENCES}

1. http://emedicine.medscape.com/article/278744-overview\#a4

2. Waddell T., Verheij M., Allum W., Cunningham D., Cervantes A., Arnold D. Gastric cancer: ESMO-ESSO-ESTRO Clinical Practice Guidelines for diagnosis, treatment and follow-up. Ann Oncol 2013; 24 Suppl 6:vi57-vi63

3. Aloe S., D'Alessandro R., Spila A., Ferroni P., Basili S., Palmirotta R., Carlini M., Graziano F., Mancini R., Mariotti S., Cosimelli M., Roselli M., Guadagni F. Prognostic value of serum and tumor tissue CA $72-4$ content in gastric cancer. Int J Biol Markers 2003; 18:21-27

4. Louhimo J., Alfthan H., Stenman U.H., Haglund C. Serum HCG beta and CA 72-4 are stronger prognostic factors than CEA, CA 19-9 and CA 242 in pancreatic cancer. Oncology 2004; 66:126-131

5. Louhimo J., Carpelan-Holmstrom M., Alfthan H., Stenman U.H., Jarvinen H.J., Haglund C. Serum HCG beta, CA 72-4 and CEA are independent prognostic factors in colorectal cancer. Int $\mathrm{J}$ Cancer 2002; 101:545-548

6. Sun Z., Zhang N. Clinical evaluation of CEA, CA19-9, CA72-4 and CA125 in gastric cancer patients with neoadjuvant chemotherapy. World J Surg Oncol 2014; 12:397

7. Emoto S., Ishigami H., Yamashita H., Yamaguchi H., Kaisaki S., Kitayama J. Clinical significance of CA125 and CA72-4 in gastric cancer with peritoneal dissemination. Gastric Cancer 2012; 15:154-161

8. Chen X.Z., Zhang W.K., Yang K., Wang L.L., Liu J., Wang L., Hu J.K., Zhang B., Chen Z.X., Chen J.P., Zhou Z.G., Mo X.M. Correlation between serum CA724 and gastric cancer: multiple analyses based on Chinese population. Mol Biol Rep 2012; 39:9031-9039

9. Spila A., Roselli M., Cosimelli M., Ferroni P., Cavaliere F., Arcuri R., Tedesco M., Carlini S., D'Alessandro R., Perri P., Casciani C.U., Greiner J.W., Schlom J., Guadagni F. Clinical utility of CA 72-4 serum marker in the staging and immediate post-surgical management of gastric cancer patients. Anticancer Res 1996; 16:2241-2247

10. Kim J.H., Jun K.H., Jung H., Park I.S., Chin H.M. Prognostic Value of Preoperative Serum Levels of Five Tumor Markers (Carcinoembryonic Antigen, CA19-9, Alpha-fetoprotein, CA72-4, and CA125) in Gastric Cancer. Hepatogastroenterology 2014; 61:863-869

11. Gwak H.K., Lee J.H., Park S.G. Preliminary evaluation of clinical utility of CYFRA 21-1, CA 72-4, NSE, CA19-9 and CEA in stomach cancer. Asian Pac J Cancer Prev 2014; 15:4933-4938
12. Safi F., Kuhns V., Beger H.G. Comparison of CA 72-4, CA 19-9 and CEA in the diagnosis and monitoring of gastric cancer. Int $\mathrm{J}$ Biol Markers 1995; 10:100-106

13. Zhang Y.H., Li Y., Chen C., Peng C.W. Carcinoembryonic antigen level is related to tumor invasion into the serosa of the stomach: study on 166 cases and suggestion for new therapy. Hepatogastroenterology 2009; 56:1750-1754

14. Ishigami S., Natsugoe S., Hokita S., Che X., Tokuda K., Nakajo A., Iwashige H., Tokushige M., Watanabe T., Takao S., Aikou T. Clinical importance of preoperative carcinoembryonic antigen and carbohydrate antigen 19-9 levels in gastric cancer. J Clin Gastroenterol 2001; 32:41-44

15. Chen S., Chen Y.B., Li Y.F., Feng X.Y., Zhou Z.W., Yuan X.H., Qian C.N. Normal carcinoembryonic antigen indicates benefit from perioperative chemotherapy to gastric carcinoma patients. World J Gastroenterol 2012; 18:3910-3916

16. Chen S., Feng X.Y., Li Y.F., Zhao B.W., Zhou Z.W., Chen Y.B. The prognosis of gastric cancer patients with marginally elevated carcinoembryonic antigen (CEA) values after D2 radical gastrectomy. J Surg Oncol 2013; 107:641-645

17. Nam D.H., Lee Y.K., Park J.C., Lee H., Shin S.K., Lee S.K., Lee Y.C., Cheong J.H., Hyung W.J., Noh S.H., Kim C.B. Prognostic value of early postoperative tumor marker response in gastric cancer. Ann Surg Oncol 2013; 20:3905-3911

18. Park H.J., Ahn J.Y., Jung H.Y., Lim H., Lee J.H., Choi K.S., Kim D.H., Choi K.D., Song H.J., Lee G.H., Kim J.H. Clinical characteristics and outcomes for gastric cancer patients aged 18-30 years. Gastric Cancer 2014; 17:649-660

19. Bagaria B., Sood S., Sharma R., Lalwani S. Comparative study of CEA and CA19-9 in esophageal, gastric and colon cancers individually and in combination (ROC curve analysis). Cancer Biol Med 2013; 10:148-157

20. Xia W., Mason A.S., Xiao Y., Liu Z., Yang Y., Lei X., Wu X., Ma Z., Peng M. Analysis of multiple transcriptomes of the African oil palm (Elaeis guineensis) to identify reference genes for RT-qPCR. J Biotechnol 2014; 184:63-73

21. Shimada H., Noie T., Ohashi M., Oba K., Takahashi Y. Clinical significance of serum tumor markers for gastric cancer: a systematic review of literature by the Task Force of the Japanese Gastric Cancer Association. Gastric Cancer 2014; 17:26-33 
22. Cainap C., Nagy V., Gherman A., Cetean S., Laszlo I., Constantin A., Cainap S. Classic tumor markers in gastric cancer. Current standards and limitations. Clujul Medical 2015; 88(2)

23. Han E.S., Lee H.H., Lee J.S., Song K.Y., Park C.H., Jeon H.M. At which stage of gastric cancer progression do levels of carcinoembryonic antigen and carbohydrate antigen 19-9 increase? Application in advanced gastric cancer treatment. $J$ Gastric Cancer 2014; 14:123-128

24. Takata A., Kurokawa Y., Fujiwara Y., Nakamura Y., Takahashi T., Yamasaki M., Miyata H., Nakajima K., Takiguchi S., Mori M., Doki Y. Prognostic value of CEA and CK20 mRNA in the peritoneal lavage fluid of patients undergoing curative surgery for gastric cancer. World J Surg 2014; 38:1107-1111

25. Laborator Synevo. Referințele specifice tehnologiei de lucru utilizate 2010. Ref Type: Catalog. 2014, cited on [01-12-2014]. Available from: https://www.synevo.ro/profil-oncologie-markeri-tumorali/

26. Li F., Li S., Wei L., Liang X., Zhang H., Liu J. The correlation between pre-operative serum tumor markers and lymph node metastasis in gastric cancer patients undergoing curative treatment. Biomarkers 2013; 18:632-637

27. Yamamoto M., Yoshinaga K., Matsuyama A., Tsutsui S., Ishida T. CEA/CA72-4 levels in peritoneal lavage fluid are predictive factors in patients with gastric carcinoma. J Cancer Res Clin Oncol 2014; 140:607-612

28. Kwon O.K., Yu W., Chung H. Prognostic value of postoperative CA19-9 normalization in patients with advanced gastric cancer. Hepatogastroenterology 2013; 60:240-243

29. Dilege E., Mihmanli M., Demir U., Ozer K., Bostanci O., Kaya C., Aksakal O., Sakiz D. Prognostic value of preoperative CEA and CA 19-9 levels in resectable gastric cancer. Hepatogastroenterology 2010; 57:674-677

30. Wang J.H., Mai C., Hong J., Zhang Q., Tang H.S., Tang Y.Q., Cui S.Z. [Predicting value of serum CEA and CA19-9 in neoadjuvant chemotherapy for advanced gastric carcinoma]. Zhonghua Wei Chang Wai Ke Za Zhi 2012; 15:1273-1276

31. Byrne D.J., Browning M.C., Cuschieri A. CA72-4: a new tumour marker for gastric cancer. Br J Surg 1990; 77:1010-1013
32. Heptner G., Domschke S., Domschke W. Comparison of CA 72-4 with CA 19-9 and carcinoembryonic antigen in the serodiagnostics of gastrointestinal malignancies. Scand J Gastroenterol 1989; 24:745-750

33. Joypaul B., Browning M., Newman E., Byrne D., Cuschieri A. Comparison of serum CA 72-4 and CA 19-9 levels in gastric cancer patients and correlation with recurrence. Am J Surg 1995; 169:595-599

34. Mittal A., Gupta S.P., Jha D.K., Sathian B., Poudel B. Impact of various tumor markers in prognosis of gastric cancer. A hospital based study from tertiary care hospital of Kathmandu valley. Asian Pac J Cancer Prev 2013; 14:1965-1967

35. Cidon E.U., Bustamante R. Gastric cancer: tumor markers as predictive factors for preoperative staging. $J$ Gastrointest Cancer 2011; 42:127-130

36. Liu X., Cai H., Wang Y. Prognostic significance of tumor markers in T4a gastric cancer. World J Surg Oncol 2012; 10:68.

37. Marrelli D., De Stefano A., de Manzoni G., Morgagni P., Di Leo A., Roviello F. Prediction of recurrence after radical surgery for gastric cancer: a scoring system obtained from a prospective multicenter study. Ann Surg 2005; 241:247-255

38. Yook J.H., Kim B.S., Kin Y.H., Suh B.S., Kim W.S., Oh S.T., Park K.C. Diagnostic Value of Tumor Markers in Stomach Cancer, J Korean Cancer Assoc 1999; 31(6): 1094-1100

39. Yamao T., Kai S., Kazami A., Koizumi K., Handa T., Takemoto N., Maruyama M. Tumor markers CEA, CA19-9 and CA125 in monitoring of response to systemic chemotherapy in patients with advanced gastric cancer. Jpn J Clin Oncol 1999; 29:550-555

40. Circulating Tumor Markers of the New Millennium. Target Therapy, Early Detection, and Prognosis. James T. Wu. Washington, DC: AACC Press, 2002, 173 pp. ISBN 1-890883-73-5

41. Fujiwara S., Wada H., Kawada J., Kawabata R., Takahashi T., Fujita J., Hirao T., Shibata K., Makari Y., lijima S., Nishikawa H., Jungbluth A.A., Nakamura Y., Kurokawa Y., Yamasaki M., Miyata H., Nakajima K., Takiguchi S., Nakayama E., Mori M., Doki Y. NY-ESO-1 antibody as a novel tumour marker of gastric cancer. Br J Cancer 2013; 108:1119-1125 